\title{
El mercado de trabajo y la feminización de la pobreza
}

Clara Ferragut Moranta

Estudiante de Ciencias Políticas y Estudios Internacionales

Universidad Carlos III de Madrid

doi: https://doi.org/10.20318/femeris.2018.4327

Las integrantes del Instituto de Estudios de Género de la Universidad Carlos III de Madrid pretenden, a través de unos seminarios interdisciplinares, analizar la situación de la mujer en el mercado laboral, especialmente la igualdad de oportunidades entre mujeres y hombres. Este proyecto ha sido financiado por el Instituto de la Mujer en la convocatoria de 2017, y se ha llevado a cabo bajo la responsabilidad de Eva Ma Blázquez Agudo, directora de la revista multidisciplinar de estudios de género Femeris. Las responsables y autoras de dicho estudio forman parte del grupo de cooperación sobre Trabajo decente y Sostenible de la Universidad Carlos III ${ }^{1}$.

En el análisis de esta cuestión y aun poniendo el foco en el marco del Estado español, cabe resaltar la relevancia internacional de dicho problema. Es decir, en la mayor parte de los Estados occidentales desarrollados las mujeres tienen unas condiciones laborales similares y se enfrentan a unas oportunidades desiguales respecto a los hombres. Estas circunstancias y oportunidades responden a unas dinámicas propias del desarrollo económico y político-social del mundo occidental. Por ello, aunque estas dinámicas parecen extenderse a lo largo del globo a través de la globalización económica y cultural, este análisis es único y no extrapolable en su totalidad a otras sociedades y culturas.

Las Naciones Unidas se hicieron eco de la situación en los Objetivos del Milenio, y lo vuelven a hacer más profundamente con los Objetivos de Desarrollo Sostenible. Los ODS son una serie de objetivos que los estados integrantes deben cumplir para asegurar un desarrollo sostenible, justo e igual de las naciones y de su ciudadanía. Así, desde el Instituto de Estudios de Género se han centrado en analizar el posible impacto de estas medidas sobre

\footnotetext{
*100349694@alumnos.uc3m.es

${ }^{1}$ Para más información consultar la web: https://www.uc3m.es/ss/Satellite/cooperacion/es/TextoDosColumnas/ $\underline{1371227819176 /}$
} 
el empleo femenino y la mejora de sus condiciones. Sin embargo, antes de ver los posibles resultados de la aplicación de los mismos, cabe ver la situación de partida. Es aquí donde la primera parte de estos seminarios, celebrado el 29 de mayo de 2018, tiene cabida. En octubre del mismo año se celebrará la segunda y última parte con el seminario titulado “Garantizando un trabajo decente a las mujeres" que concluirá este análisis interdisciplinar.

A través de siete expertas y expertos en su ámbito, y de cinco ponencias, es posible hacerse una idea clara de cuáles son los influjos y marcos que afectan a la situación de la mujer en el mercado laboral. A continuación, se presenta un resumen de cada ponencia para que la lectora y el lector puedan conocer mejor los factores y las dinámicas que juegan en este mercado.

\section{Daniel Pérez del Prado y el contexto socioeconómico de la inserción de las mu- jeres en el marco de los ODS}

Daniel Pérez del Prado, licenciado en derecho y economía, y doctor en derecho, es el coordinador de este primer seminario. En su ponencia enmarca la problemática y el análisis en perspectiva de género desde el punto de vista socio-económico. El ponente centra el contexto de partida en relación con los objetivos 5) Igualdad de Género, y 8) Trabajo decente y crecimiento económico. Los indicadores que resaltan y los principales resultados son los siguientes:

a) "La proporción de tiempo dedicado al día al trabajo doméstico y de cuidados no remunerado". En España, y en concordancia con la media europea, existencia una diferencia de unos once puntos, siendo la dedicación de las mujeres superior.

b) "La tasa de actividad". Oceanía, Europa, América del Norte y África Subsahariana son las zonas que presentan una menor diferencia por géneros en 2017.

c) "La brecha salarial". En 2016 la media de la UE se situaba en 16,2 puntos, y la brecha española en 14.2 puntos. El país europeo con una menor brecha salarial es Rumania.

d) "La tasa de desempleo". España es el segundo país de la UE, en 2017, con una mayor tasa de desempleo y con la mayor diferencia entre desempleo femenino $(19,2)$ y masculino $(15,7)$. La crisis tuvo un efecto coyuntural que hizo que las tasas por género confluyeran en un momento. Sin embargo, a medida que se están superando los efectos de la crisis la tasa de desempleo femenino ha vuelto a ser superior.

e) "La tasa de parcialidad". Tanto en España como en el conjunto de la UE la parcialidad es predominantemente femenina. Los datos muestran una mayor incidencia femenina en la parcialidad en Europa que en España, sin embargo, estos datos están sesgados por dos razones principales. Primero, en España son más comunes los contratos temporales que a tiempo parcial. Segundo, España no computa la reducción de jornada. Es decir, el tipo de contrato no cambia jurídicamente con lo que sigue figurando y computando estadísticamente como un contrato a tiempo 
completo cuando tanto las horas como el salario corresponden a un contrato de tiempo parcial.

\section{Miguel García-Cesto Romero y el contexto socio-demográfico de la inserción de la mujer en el trabajo fuera del hogar}

Miguel García-Cesto licenciado en Derecho y Ciencias Económicas, y es actualmente el director de ASAP operational research. Este ponente completa los datos anteriores desde el punto de vista demográfico.

García-Cesto relaciona la crisis de trabajo con el crecimiento poblacional. Debido a que las economías actuales cada vez necesitan menos horas de trabajo humano para mantener la productividad, el aumento de la población crea desempleo porque las economías, explica el ponente, son incapaces de absorber la mano de obra. Define el mercado de trabajo como un entorno hostil debido a la diferencia entre la oferta y la demanda, y la sustitución del trabajo humano por maquinaria. En este contexto las mujeres se ven especialmente perjudicadas debido a que son las últimas en entrar en el mercado laboral y porque siguen asumiendo las labores de cuidados. Esto está claramente relacionado con la estructura familiar española.

Una clara consecuencia de esta situación es que las mujeres se ven obligadas a elegir entre la maternidad o una carrera profesional próspera. Esto provoca una disminución de la tasa de maternidad y a largo plazo, una sociedad cada vez más elitista, ya que las familias con recursos económicos abundantes son aquellas que pueden compaginar ambas cosas, externalizando las tareas de cuidados a mujeres, especialmente migrantes.

\section{Cristina Aragón Gómez y Maravillas Espín Sáez con la igualdad en el acceso a los recursos económicos}

Cristina Aragón es licenciada en Derecho, complementa su carrera con el máster en Dirección de Recursos Humanos y otro en Derecho Privado, ámbito con el cual obtiene su doctorado y desarrolla su actividad. Por otro lado, Maravillas Espín también es licenciada en derecho y doctorada en la misma área. Ambas se han desarrollado profesionalmente en el Derecho del Trabajo y de la Seguridad Social.

Estas dos ponentes se centran en la disparidad de los recursos económicos por género en los trabajos asalariados y en el trabajo por cuenta propia. En cuanto a los primeros, la profesora Aragón expone que las mujeres tienen mayores dificultades para acceder a este y cuando lo hacen, suelen tener unas condiciones más precarias (mayor temporalidad, mayor parcialidad y salarios más bajos). Destaca que existen unas discriminaciones sociales prácticamente invisibles que afectan a la brecha salarial y por ende a los recursos económicos personales. Por ejemplo, la edad, la segregación ocupacional (peor valoración social de los trabajos feminizados y menor ocupación de los puestos directivos), o la asunción de las responsabilidades de cuidados. La brecha salarial en España responde a otros 
factores más a parte de las horas trabajadas, ya que cuando se mira la ganancia media por hora independientemente del contrato sigue existiendo esta brecha.

En cuanto al acceso a los recursos por cuenta propia, es importante destacar que más de la mitad de los trabajadores autónomos son varones y muchas mujeres cumplen con el papel de colaboradoras. La mayoría de mujeres autónomas tienen microempresas o bien no ocupan a ningún trabajador.

Esta imagen nos muestra una dificultad en el acceso a los recursos económicos por parte de las mujeres. Un claro y muy significativo efecto de esto es el acceso a las prestaciones de la seguridad social. En el sistema contributivo español la cuantía prestacional está íntimamente relacionada con las bases reguladoras. En consecuencia, el acceso más dificultoso y unas condiciones más precarias hacen que las mujeres contribuyen menos y por ende tengan dificultades para acceder a las prestaciones y cuando acceden la cuantía que reciben es menor. A día de hoy existe una brecha de género enorme en las prestaciones.

\section{Patricia Nieto Rojas y Pablo Gimeno con la flexibilidad de las condiciones labo- rales como elemento de integración de la trabajadora}

Patricia Nieto Rojas, es diplomada en Relaciones Laborales y licenciada en Ciencias del Trabajo, tiene un máster en Derecho Privado y es doctorada con una tesis sobre la "representación de los trabajadores en la empresa". Pablo Gimeno es licenciado en Economía y Derecho y Doctor en Derecho. Ambos desarrollan su actividad investigadora y académica en el marco de mercado de trabajo.

Esta ponencia se centra en el binomio trabajo decente y empleo femenino. Analizan la precarización de la mujer dentro del mercado laboral en referencia al tiempo y lugar de trabajo. A grandes rasgos uno de los factores que envuelven a las mujeres en su desarrollo profesional es la perpetuación del rol de mujer cuidadora y en su aspecto moderno, el rol de mujer migrante contratada -a través la economía sumergida- para llevar a cabo ese rol. Cuando las mujeres entran en el mercado de trabajo, los hombres no entran en el mercado de cuidados, lo que provoca una disminución del tiempo disponible de las mujeres y de su flexibilidad, además de una doble jornada laboral. Las mujeres y madres tienen una menor capacidad de movilización social debido a un mercado de trabajo que no concilia mujer trabajadora y maternidad. Las mujeres en el mercado de trabajo español están penalizadas no solo por razón de género sino especialmente por ser madres. Patricia Nieto destaca que el "elemento que más influye a despenalizar las mujeres es el permiso intransferible para ambos géneros". El empresario aquí no puede discriminar ya que se ve en la obligatoriedad de considerar a ambas personas con la misma carga.

La ponente destaca la importancia de cada contexto a la hora de llevar a cabo el análisis y pensar en las propuestas. Por ejemplo, se habla de flexibilizar la jornada laboral e implementar el trabajo por objetivos. Para los trabajos de oficina o comerciales son medidas muy efectivas, sin embargo, no hay que olvidar que estos no son aplicables a otros ámbitos laborales. 


\section{María Gema Quintero y los colectivos vulnerables por razón de género en el mer- cado laboral}

María Gema Quintero es licenciada en Derecho y Doctora en Derecho. Desarrolla su actividad investigadora en el ámbito de la protección social en materia de Seguridad Social.

La profesora Quintero habla sobre los elementos de vulnerabilidad en el empleo. Los parámetros cuantificables son la inactividad, el desempleo y la precariedad laboral. Estos difieren si se analizan desde la perspectiva individual o desde la perspectiva colectiva, el hogar como unidad económica.

En todas ellas, el género es una condición personal de vulnerabilidad, pero también es la base de otros factores de vulnerabilidad. Pueden ser por ejemplo la edad, la discapacidad, la nacionalidad, el nivel formativo, el lugar de residencia, etc.

Cuando se observan los datos por el tipo de sector productivo, este también se convierte en un factor de vulnerabilidad al estar las mujeres más representadas en aquellos sectores más precarios.

La conclusión a la que llega la ponente, analizando todos estos factores, es que, dentro de cada colectivo vulnerable, el género es un factor exponencial de vulnerabilidad. Es decir, dentro de cualquier sector precario, edad vulnerable, nacionalidad discriminada o estigmatizada, nivel de formación existe una escala jerárquica de hombre - mujer. 